\title{
How I do VATS segmentectomy: the two-port approach
}

\author{
Alessandro Brunelli \\ Department of Thoracic Surgery, Leeds Teaching Hospitals NHS Trust, Leeds, UK \\ Correspondence to: Alessandro Brunelli. Department of Thoracic Surgery, St. James's University Hospital, Leeds, UK. Email: brunellialex@gmail.com.
}

\begin{abstract}
The authors describe their bi-portal videoassisted thoracoscopic approach to perform anatomic segmentectomies. Operating room setting and details of individual segmentectomies performed through this approach are described. The approach consists of a $3-4 \mathrm{~cm}$ anterolateral utility incision performed at the level of 4th or 5th intercostal space and another 1-1.5 cm incision at the level of the 6-7th intercostal space performed just anterior to the posterior axillary line. In general, a wound protector is applied in the utility incision and a trocarless technique is used in the inferior port. The utility and the inferior incisions are used often interchangeably and the type and sequence of instrument introduced through one or the other port change depending on the type of segmentectomy. In general, for upper lobe segmentectomies, the thoracoscope is introduced in the utility incision, while for lower lobe segmentectomies it is positioned in the inferior port. All procedures start with lung inspection to identify the position of the tumour in relation to the preoperative imaging. A pre-emptive intercostal analgesia is performed. Subsequently, the segmentectomy procedure is performed with individual dissection and division of the segmental hilar structures and final division of the intersegmental plane. A systematic lymph node dissection is always performed.
\end{abstract}

Keywords: Segmentectomy; sublobar resections; video-assisted thoracoscopic surgery (VATS)

Received: 16 May 2021; Accepted: 05 July 2021; Published online: 28 July 2021.

doi: 10.21037 /jovs-21-24

View this article at: https://dx.doi.org/10.21037/jovs-21-24

\section{Introduction}

The two-port approach for anatomic lung resection was first described by Iwasaki and coll. (1) and then popularised by the Duke University team (2).

It consists of an anterior approach including a $3-4 \mathrm{~cm}$ anterolateral utility incision performed at the level of 4th or 5 th intercostal space and another $1-1.5 \mathrm{~cm}$ incision at the level of the 6-7th intercostal space performed just anterior to the posterior axillary line (Figures 1,2).

In general, a wound protector is applied in the utility incision and a trocarless technique is used in the inferior port (if the camera is positioned in this port the trocar is retracted back after its introduction to minimise trauma to the intercostal nerve).

The approach is standard for all types of lobectomies or segmentectomies and for both sides.

As a rule, I tend to perform first the utility incision and then after inspecting the pleural cavity with the camera selecting the best level to perform the second port.

The utility and the inferior incisions are used often interchangeably and the type and sequence of instrument introduced through one or the other port change depending on the type of segmentectomy.

Our indications for a segmentectomy include the followings: (I) intentional segmentectomies (performed in patients fit for lobectomy with a peripheral tumour smaller than $2 \mathrm{~cm}$, with sufficient margins anticipated in the preoperative planning); (II) compromise functional segmentectomy (in patients deemed at too high risk for a lobectomy); (III) compromise diagnostic segmentectomy (in patients with undiagnosed small tumours, in whom computed tomography (CT) guided biopsy or wedge is not feasible, or navigational bronchoscopy did not yield diagnostic results, or with a low malignancy score, or with a history of previous tumours in other organs hinting at the possibility of metastasis); (IV) compromise multiple (in patients with synchronous or metachronous tumours 


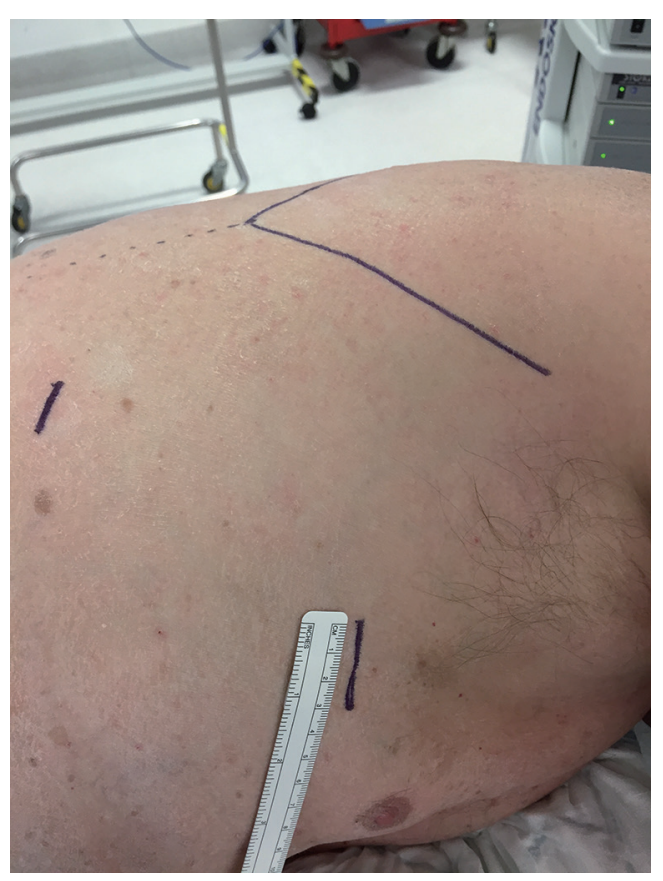

Figure 1 Right biportal VATS approach. VATS, video-assisted thoracoscopic surgery.

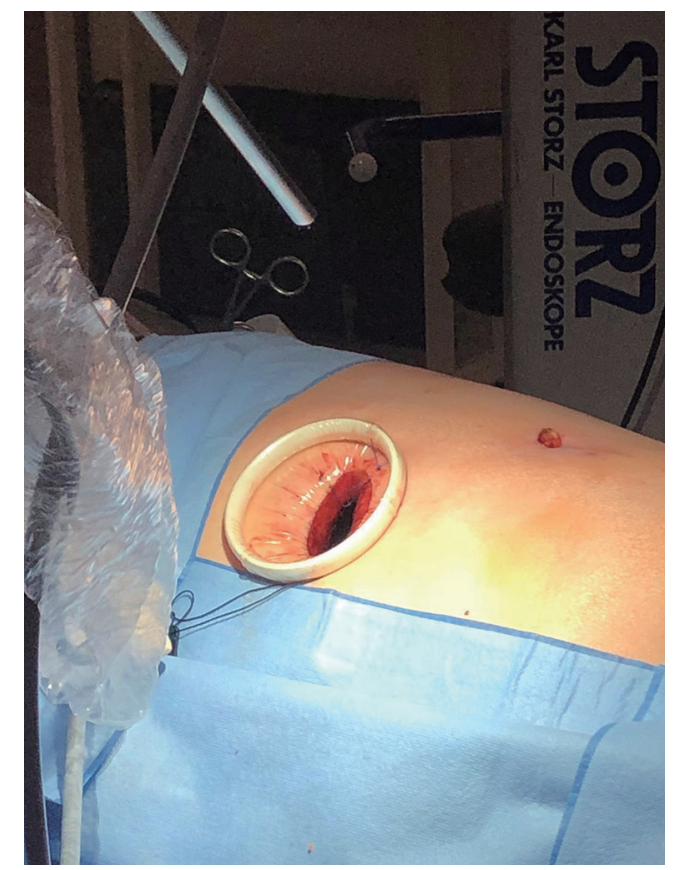

Figure 2 Left biportal VATS approach. VATS, video-assisted thoracoscopic surgery.

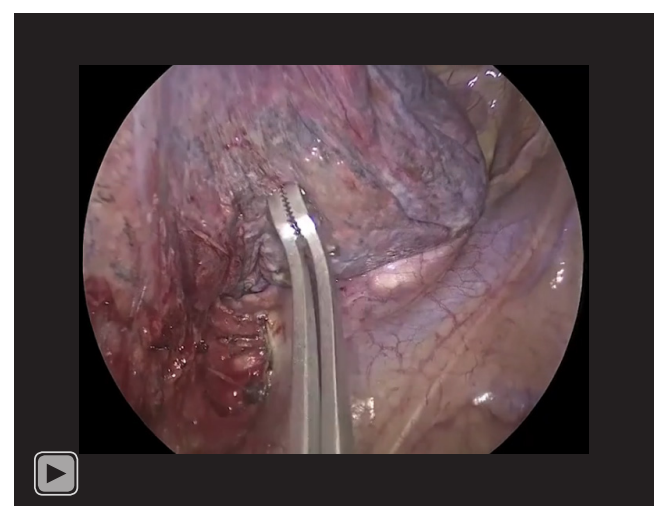

Video 1 Right biportal VATS apical segmentectomy (right S1).

or who underwent a previous lung resection and in whom sparing parenchyma appears convenient).

\section{Case presentation}

\section{Initial phase (common for all segmentectomies)}

A $10 \mathrm{~mm} \mathrm{30-degree} \mathrm{scope} \mathrm{is} \mathrm{introduced} \mathrm{through} \mathrm{the}$ utility incision. The lung is inspected. The second port is performed. The camera is then swapped to the inferior port. A pre-emptive analgesia using multilevel intercostal blocks from the 2 nd through the last visible intercostal space is performed utilising a combination of drugs $(20 \mathrm{~mL}$ bupivacaine $0.5 \%$ and adrenaline 1:200,000, dexamethasone $10 \mathrm{mg}$ and clonidine $150 \mathrm{mcg}$ ).

The lung is inspected and the tumour is identified and if needed marked.

At this stage, the steps will differ depending to the type of segmentectomy.

\section{Apical and anterior segmentectomies of right upper lobe; anterior segment of the left upper lobe (Video 1)}

The two surgeons are positioned on the same side of the patient, anteriorly (Figure 3). The camera is placed in the utility incision. A suture is placed in the upper corner of the utility incision to suspend the camera as a hammock (Figure 4A,4B). This makes the work of the assistant more ergometric. The vision from the utility incision is the same as in the uniportal approach and open surgery. The inferior port is used to pass a lung grasper and retract the lung. We generally use a $350 \mathrm{~mm}$ long lung grasper with a $10 \mathrm{~mm}$ shaft diameter. In general, the segmental hilum dissection is performed using instruments introduced from the utility 


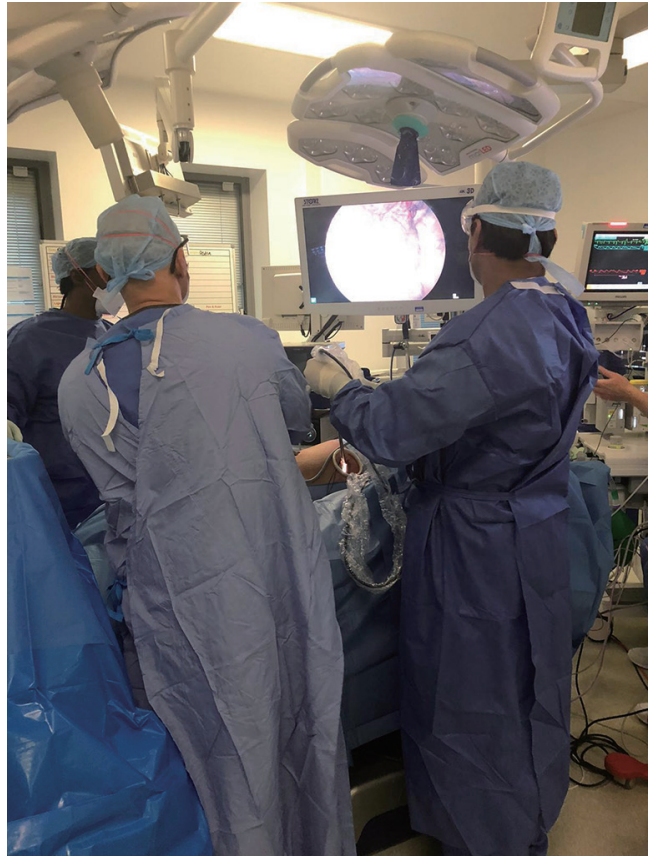

Figure 3 Operating room setting in a typical case of upper lobe segmentectomy.

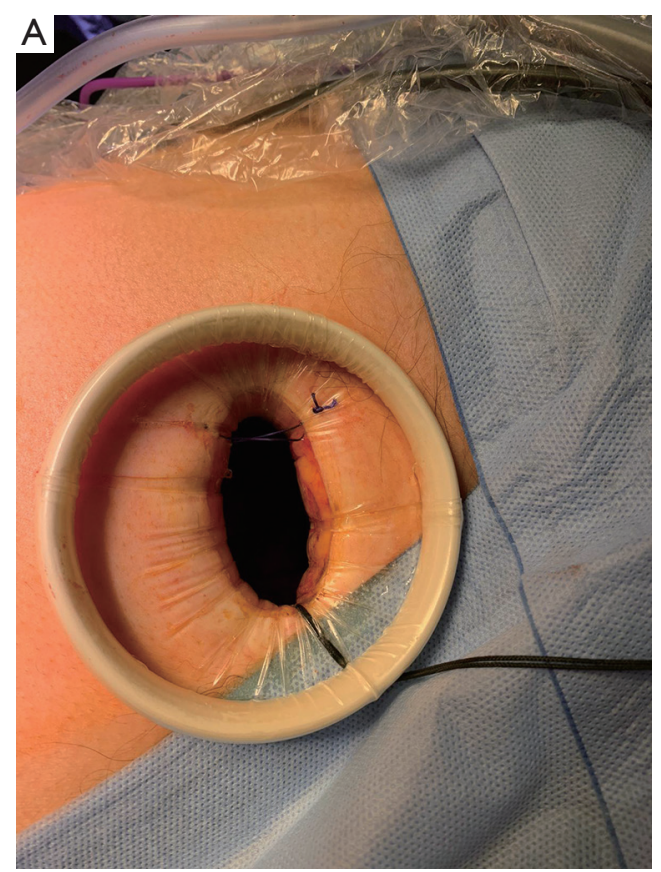

incision (a combination of suction device, energy device and cautery, DeBakey dissector-330 mm long with a $7-10 \mathrm{~mm}$ shaft diameter). The endo staplers (EndoGIA Covidien or Echelon Johnson \& Johnson) to divided vessels, segmental bronchus and the intersegmental planes are introduced either from the utility incision or the inferior port according to the best angle of exposure.

The intersegmental plane is generally divided along the inflation deflation line after selective clamping of the segmental bronchus and re-ventilation of the lung. In most of the segmentectomies however the determination of the intersegmental plane line is assisted by the direction of the divided segmental hilar structures and most of all the remaining hilar vessels to preserve.

We usually strive to ensure at least $1-2 \mathrm{~cm}$ free margin in intentional segmentectomies. However, in compromise segmentectomies where the tumour may be bigger and there is a particular functional need to preserve as much parenchyma as possible this limit may be lower.

\section{Technical pearls}

In the right apical segmentectomy pay particular attention

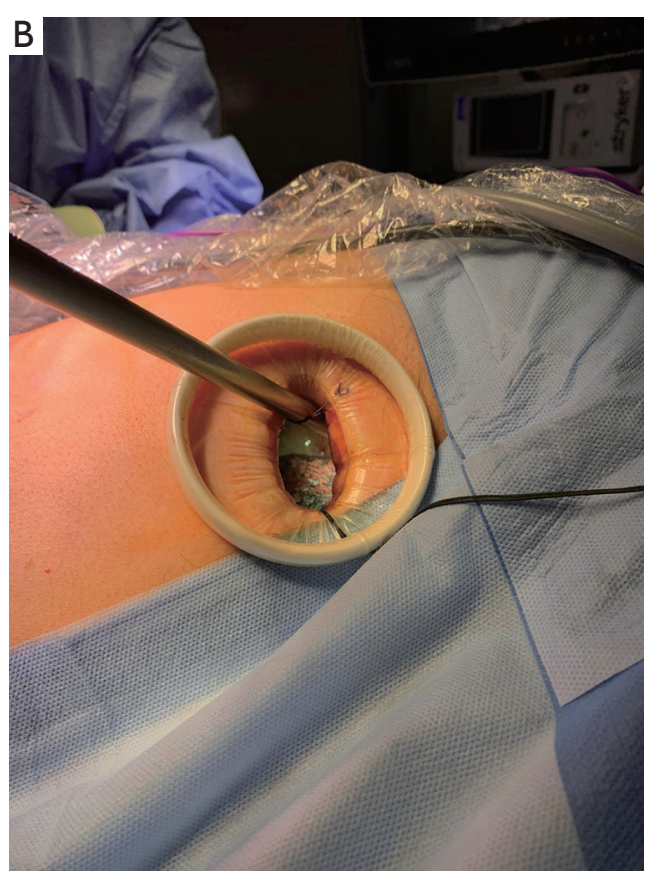

Figure 4 A suture is placed in the upper corner of the wound protector to suspend the thoracoscope and facilitate the work of the assistant. (A) Suture is placed in the upper corner of the wound protector; (B) this suture is used to suspend the thoracoscope and facilitate the work of the assistant. 


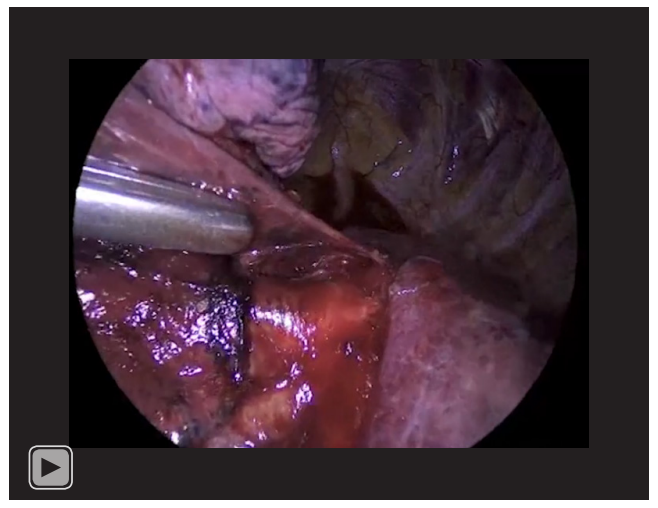

Video 2 Left biportal VATS apico-posterior segmentectomy (left $\mathrm{S} 1+\mathrm{S} 2)$.

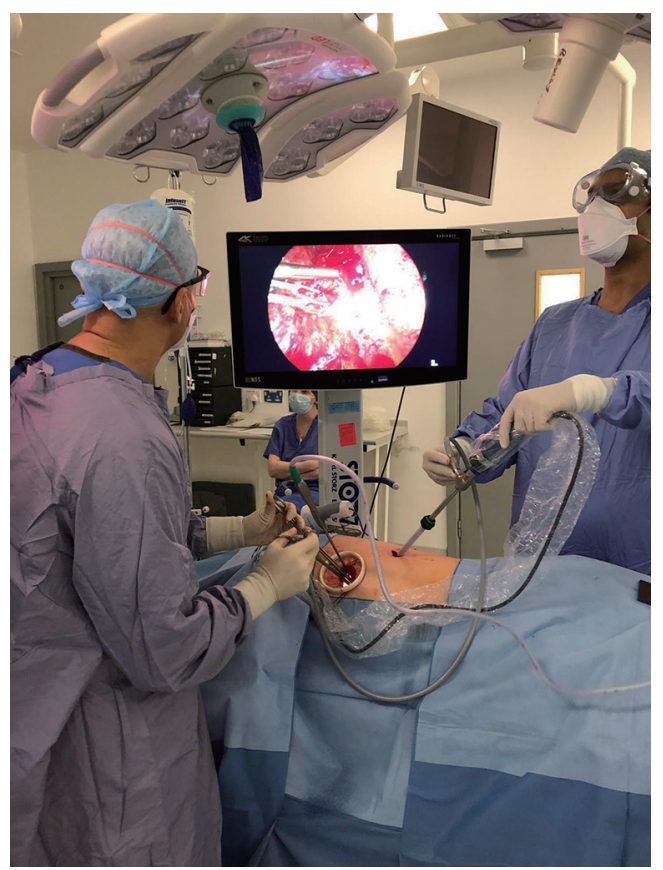

Figure 5 Operating room setting in a typical case of lower lobe segmentectomy.

to the dissection of the B1 segmental bronchus (after division of the A1 artery). The instrument used to dissect the bronchus deep and peripheral into the parenchyma and introduced from the utility incision may stretch and compress the A3 anterior branch of the PA which consequent risk of vascular injury. In addition, the A1 segmental branch should be divided distal to the origin of the A2 branch. To this purpose, this segmental vessel should be isolated very peripherally.
In the right anterior segmentectomy, a very convenient step is the early completion of the horizontal fissure once the V3 anterior branch of the superior pulmonary vein has been divided and the pulmonary artery identified behind the pulmonary vein. The division of the horizontal fissure facilitate the subsequent exposure of the segmental hilar vessels and bronchus.

\section{Posterior segment of right upper lobe and apico-posterior segment of left upper lobe (Video 2)}

To approach these segments, the camera is usually placed in the inferior port (Figure 5). This provides an excellent visualization of the fissure. The surgeon is always positioned in front of the patient and the assistant is placed on the other side of the table (back of the patient). The instruments utilized to dissect the segmental vessels and bronchus are introduced instead from the utility incision. Similarly, the endostaplers to divide the segmental hilar structures and intersegmental planes are also introduced from the utility incision, although in some circumstances and for difficult angles the staplers are also introduced from the inferior port.

In these segmentectomies an important step is the preliminary dissection of the posterior mediastinum with station 7 lymphadenectomy and exposure of the secondary carina, which facilitates the further steps of the procedure. This initial step is performed with the camera placed in the inferior port and the dissecting instruments introduced from the utility incision.

\section{Technical pearls}

Both these segmentectomies require an intrafissural dissection of the segmental arteries and bronchi. Whereas the segmental vein is generally identified at this level for right S2 segmentectomies, division of the vein is not generally performed in left $\mathrm{S} 1+2$ segmentectomies. For the left $\mathrm{S} 1+2$ segmentectomies the lingular branch of the PA should be identified and spared. This branch represents the lower level to start the intersegmental plane division.

\section{Lingulectomy}

The relative position of the thoracoscope and instruments in the lingulectomy will depend on the completeness of the interlobar fissure. The two surgeons are usually both standing on the frontal side of the patient. In general, the lingular vein is best visualised placing the camera in the utility incision and divided passing the dissector and 


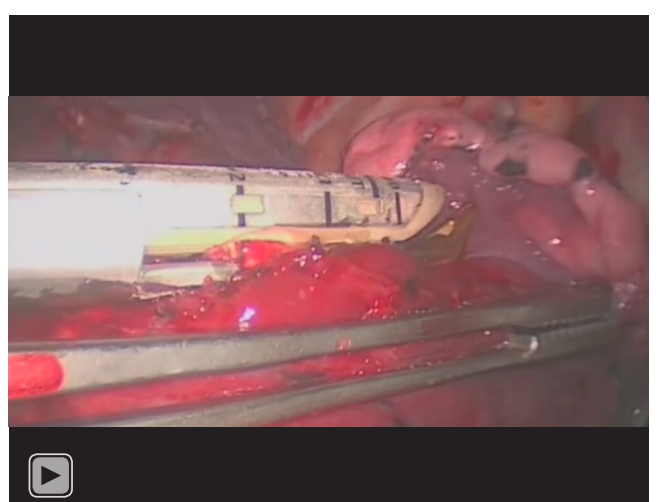

Video 3 Left biportal VATS posterior-lateral basal segmentectomy (LS9-10).

endostapler through the inferior port. Then the camera can be swapped into the inferior port to provide an excellent visualization of the fissure from below and the lingular branches of the pulmonary artery and lingular bronchus which need to be isolated and then divided with instruments and staplers usually introduced from the utility incision. Also, the intersegmental plane is generally divided with staplers introduced from the utility incision although like in every segmentectomy, the other port can be used if felt to provide a better angle.

\section{Superior segmentectomy and other basilar segmentectomies of lower lobes (Video 3)}

In this segmentectomy, like for the other segmentectomies of the lower lobe the thoracoscope is generally held in the inferior port and the dissecting instruments are introduced from the utility incision. The surgeon stands in front of the patient and the assistant remains on the other side (back of the patient). This will hold true for both fissure-last and fissure first approach. However, during some steps of the procedure it may be more convenient to move the camera in the utility incision to have a more direct visualization of the fissure in case of intrafissural approach and incomplete fissure. The first step of every lower lobe segmentectomy is the dissection of the posterior mediastinum and station 7 lymphadenectomy which will expose posteriorly the hilum of the lung up to the secondary carina.

The most complex part of the lower lobe segmentectomies is the division of the intersegmental plane, especially in case of single basilar segmentectomies. In this case the thoracoscope can be moved from the inferior port to the utility and the endo staplers passed through the inferior port if this is considered a better angle of division.

\section{Technical pearl}

For segmentectomies 9 and 10, we use either an intrafissural approach when the fissure is complete and divide the superior segment of the lower lobe from the basilar segments to simplify the subsequent intersegmental plane division (3). In case the fissure is not complete we have sometime used a fissureless approach by dividing the segmental vein first and then the segmental bronchus and artery from the posterior aspect of the hilum. Occasionally it may be more convenient to keep the camera in the inferior port and introduce the staplers along the camera to have a synchronised vision.

\section{Lymphadenectomy}

As mentioned before, station 8,9 and subcarinal lymphadenectomies (station 7) are generally performed with the thoracoscope in the inferior port and the dissecting instruments (suction, nodal grasper, energy device or cautery) introduced from the utility incision.

Other stations such as right paratracheal or station 5 and 6 on the left side are generally approach with the camera in the utility incision and instruments also introduced from the same utility incision.

Care must be taken to remove the lymph nodes from the utility incision covered with a wound protector to prevent tumour seeding of the surgical wound.

\section{Final steps of the operation}

The specimen is removed using an endo-bag through the utility incision. The hilum and nodal stations are inspected to check any bleeding.

The lung is checked for any air leak from the segmental plane or segmental bronchial stump using a submersion test. Finally, a single $24 \mathrm{Ch}$ chest drain is inserted through the inferior port and the lung is re-expanded under vision. The tube is positioned posteriorly up to the apex after most of the segmentectomies, with the exception of the anterior segmentectomies and lingulectomy in which the tube is positioned in a midline lung position up to the apex.

All procedures performed in this study were in accordance with the ethical standards of the institutional and/or national research committee(s) and with the Helsinki Declaration (as revised in 2013). Written informed consent 
was obtained from the patient for publication of this manuscript and the accompany images and videos. A copy of the written consent is available for review by the editorial office of this journal.

\section{Discussion}

We presented our surgical approach technique to minimally invasive anatomic segmentectomies which consists of a two ports anterior approach. We think this technique is simple to learn, safe and represent a valuable alternative to threeport or single port approaches. One of the main features of this approach is the use of the thoracoscope from the utility incision for the upper resections which provides a similar exposure and perspective as in the open approach. In addition, the use of a second inferior small access allows for more flexibility in introducing instruments to retract the lung or staplers especially for upper lobe resections with a more advantageous angle with respect to single port approach.

One important tip is to avoid the use of trocar in the inferior port to minimise trauma to the intercostal nerve. Similarly leveraging of the camera or any instrument in the port should be avoided for the same reason.

The availability of a second small incision prevents also the crowding of instruments in the utility incision facilitating the work of the surgeon and minimising once again trauma to the intercostal nerve at this level.

The single chest drain is inserted through the inferior port and no additional incision is required.

The availability of the inferior port 9 which is located more posteriorly with respect of the utility incision) facilitates also the exposure of the posterior mediastinum for the subcarinal lymphadenectomy and any subsequent posterior dissection.

\section{Acknowledgments}

Funding: None.

\section{Footnote}

Provenance and Peer Review: This article was commissioned by the Guest Editor (Michel Gonzalez) for the series "VATS Segmentectomy" published in Fournal of Visualized Surgery. The article has undergone external peer review.

Conflicts of Interest: The author has completed the ICMJE uniform disclosure form (available at https://jovs. amegroups.com/article/view/10.21037/jovs-21-24/coif). The series "VATS Segmentectomy" was commissioned by the editorial office without any funding or sponsorship. The author declares consulting fees from Medtronic, Astra Zeneca, Ethicon and BD; and payment or honoraria from Ethicon, Medtronic, and Astra Zeneca; and serves as an unpaid editorial board member of Fournal of Visualized Surgery from June 2019 to May 2021. The author has no other conflicts of interest to declare.

Ethical Statement: The author is accountable for all aspects of the work in ensuring that questions related to the accuracy or integrity of any part of the work are appropriately investigated and resolved. All procedures performed in this study were in accordance with the ethical standards of the institutional and/or national research committee(s) and with the Helsinki Declaration (as revised in 2013). Written informed consent was obtained from the patient for publication of this manuscript and the accompany images and videos. A copy of the written consent is available for review by the editorial office of this journal.

Open Access Statement: This is an Open Access article distributed in accordance with the Creative Commons Attribution-NonCommercial-NoDerivs 4.0 International License (CC BY-NC-ND 4.0), which permits the noncommercial replication and distribution of the article with the strict proviso that no changes or edits are made and the original work is properly cited (including links to both the formal publication through the relevant DOI and the license). See: https://creativecommons.org/licenses/by-nc-nd/4.0/.

\section{References}

1. Iwasaki M, Kaga K, Nishiumi N, et al. Experience with the two-windows method for mediastinal lymph node dissection in lung cancer. Ann Thorac Surg 1998;65:800-2.

2. Burfeind W R, D'Amico T A. Thoracoscopic lobectomy. Operat Tech Thorac Cardiovasc Surg 2004;9:98-114.

3. Igai H, Kamiyoshihara M, Kawatani N, et al. Thoracoscopic lateral and posterior basal $(\mathrm{S} 9+10)$ segmentectomy using intersegmental tunnelling. Eur J Cardiothorac Surg 2017;51:790-1.

doi: 10.21037/jovs-21-24

Cite this article as: Brunelli A. How I do VATS segmentectomy: the two-port approach. J Vis Surg 2023;9:14. 